\title{
TH-VSS: An Asymmetric Storage Virtualization System for the SAN Environment
}

\author{
Da Xiao, Jiwu Shu, Wei Xue, and Weimin Zheng \\ Department of Computer Science and Technology, Tsinghua University, \\ 100084 Beijing, China \\ xiaoda99@mails.tsinghua.edu.cn
}

\begin{abstract}
Storage virtualization is a key technique to exploit the potential of SANs. This paper describes the design and implementation of a storage virtualization system for the SAN environment. This system has asymmetric architecture, and virtualization operations are done by a metadata server allowing management to be accomplished at a single point. The system has better scalability compared to symmetric systems and can support heterogeneous platforms of hosts. Agent software was implemented in the volume management layer of hosts so that any standard HBA card can be used. The metadata server manages storage resources automatically and configuration of storage pools can be changed dynamically. The metadata server is also used for system monitoring, which is the basis of dynamic storage resource management and automatic failure recovery. Test results showed that the overhead introduced by our virtualization layer is negligible, and the performance of storage system was enhanced effectively by the striping strategy in organizing storage pools. The number of seeks per second to a logical volume allocated from a pool of four disks was increased by $55.2 \%$ compared to a plain FC disk.
\end{abstract}

\section{Introduction}

The introduction of storage area networks (SANs) can significantly improve the reliability, availability, and performance of storage systems. However, SANs must be managed effectively so that their potential can be fully exploited. Storage virtualization is often seen as the key technology in SAN management. Storage virtualization separates physical storage from the server's operating system and provides storage users with unified storage pools and logical volumes. It can provide users with much larger storage space than a single physical disk and a much better utilization of disk capacity. Furthermore, virtualization offers a new level of flexibility. Storage systems can be added to or removed from storage pools without downtime, thus enabling fast adaptation to new requirements.

The first approach to storage virtualization in a cluster environment is hostbased. Two typical examples of this approach are CLVM[3] and the EVMS cluster[5]. CLVM is an extension of Linux LVM in a single system environment to add cluster support. The EVMS cluster is a framework proposed by IBM which 
also supports virtualization in cluster environments. Both of these approaches adopt a host-based symmetric architecture. Each node in the cluster has the right to perform virtualization management tasks. The consistency of metadata is maintained by communications between nodes. This puts a heavy burden on hosts and reduces the scalability of the system. Besides, heterogeneous platforms of hosts are not supported by systems with this symmetric architecture.

Another approach is network-based in-band virtualization. This approach calls for an appliance to be installed between the hosts and the storage devices, which redirects all I/Os between hosts and storage devices. Representatives of this approach are DataCore Software's SANsymphony[6] and HP's StorageApps[4]. The advantage of this approach is that agent software need not be installed on hosts, so transparency to hosts is achieved. Its main drawback is that the appliance may become a SAN bottleneck, thus limiting the SAN performance and scalability and significantly complicating the design of large-scale highly available configurations.

A third approach is network-based out-of-band virtualization. In this approach, the handling of metadata is separated from the data path and is done by a dedicated appliance. It enables direct data transfer between hosts and storage subsystems. StoreAge's SVM[7] adopts such an architecture.

Moreover, the main consideration in all the approaches mentioned above is storage capacity when grouping physical disks into storage pools. These approaches ignore other properties of virtual storage such as bandwidth, latency, and reliability.

This paper describes the design and implementation of a storage virtualization system for SAN environments called the Tsinghua virtualization storage system (TH-VSS). In this system, an asymmetric architecture is adopted. Metadata management tasks are done by a metadata server so that single-point management is achieved. The system has better scalability compared to symmetric virtualization architecture and can support heterogeneous platforms in hosts. Agent software is implemented in the volume management layer of hosts, which results in improved flexibility. Any standard HBA card can be used. Storage resources are managed automatically by the metadata server, and the configuration of storage pools can be changed dynamically. The metadata server is also used for system monitoring, which is the basis of dynamic storage resource management and automatic failure recovery.

\section{TH-VSS Design}

\subsection{Architecture of TH-VSS}

TH-VSS consists of three parts: the virtualization agents on the hosts, a meta server, and storage devices. The hosts and meta server are attached to the SAN via a FC HBA on the motherboard. Storage devices are also attached to the SAN via their fibre channel interfaces. The meta server and agent are connected to each other through Ethernet. The TH-VSS was designed for a heterogeneous 


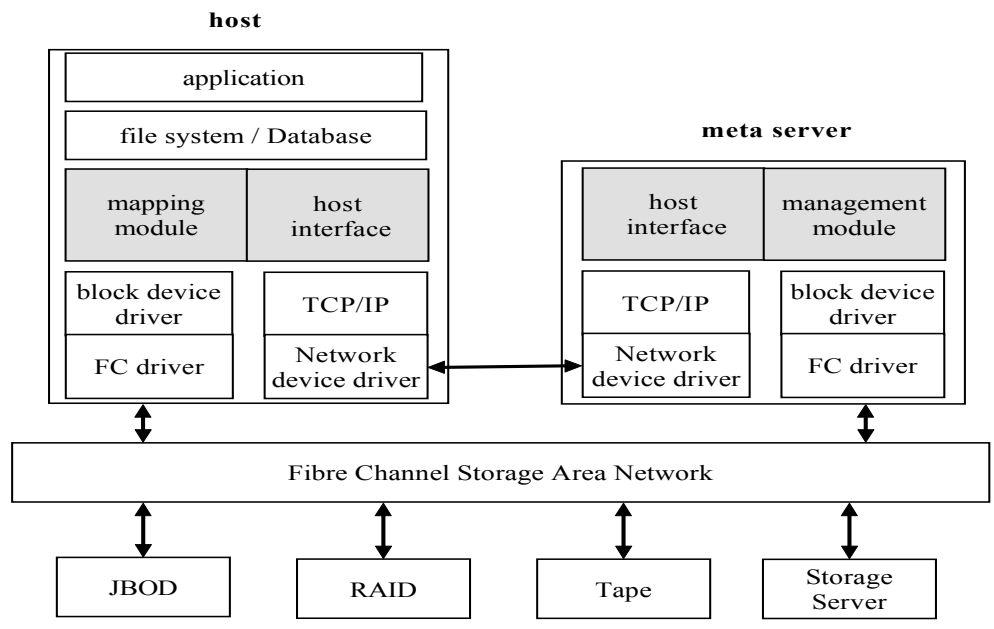

Fig. 1. Overall architecture of the TH-VSS

SAN environment. Hosts may run different OSs, and storage devices include JBOD, RAID subsystems, tape drives, and storage servers, as well as others. The metadata management module on the meta server performs the metadata management tasks, and logical to physical address mapping of I/O requests are handled by the mapping layer of the agent on the host. The software architecture of the system is shown in Fig. 1 (gray boxes are software modules implemented in the TH-VSS).

\subsection{Virtualization Process}

The system works as shown in Fig. 2. On startup, the system monitor on the meta server scans all physical storage devices attached to the SAN. For each device, the system monitor writes a label and a UUID on the device to create a physical volume on it. Then the system monitor groups the storage devices into different storage pools according their detected properties. Next, the administrator issues a command to create a logical volume through the administration interface. The administration interface tells the metadata manager to allocate space for the logical volume from the storage pool and create it. Then the administrator issues another command to assign the volume to the Solaris server. On receiving such a command, the meta server sends the UUIDs of the physical volumes in the storage pool and the mapping table of the volume to the agent on the Solaris server through host interfaces. The agent locates the correct disks according to the UUIDs and creates the logical volume in a kernel for further use. Then it sends a response to the meta server to tell it that the volume has successfully been created. Finally, applications on the Solaris server can access the logical volume directly without consulting the meta server. 


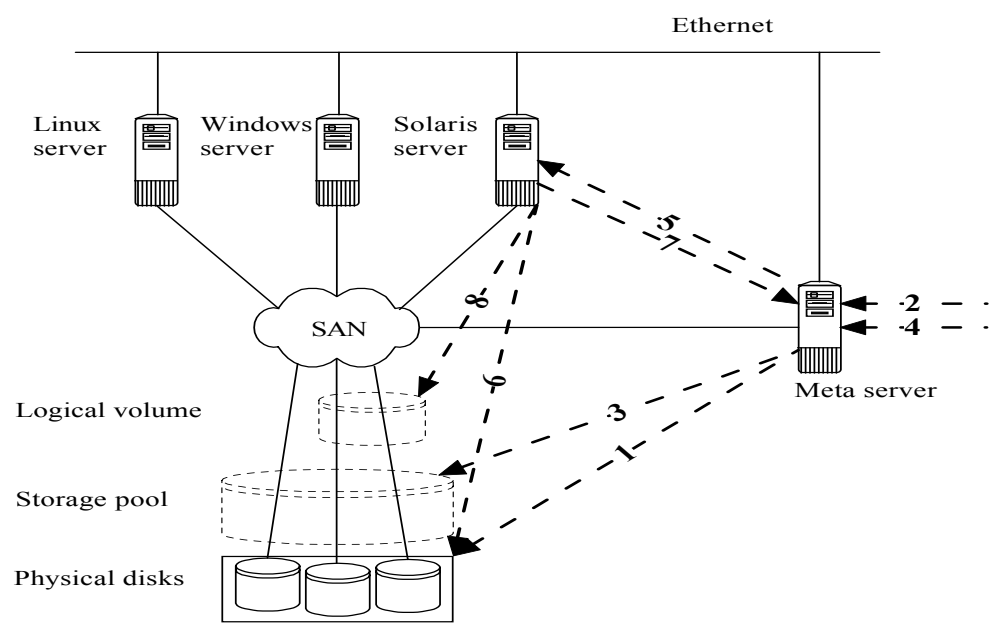

Fig. 2. Virtualization process

\section{Key Techniques in TH-VSS Implementation}

\subsection{Storage Resource Management}

The lowest level in the TH-VSS storage hierarchy is the physical volume. Multiple physical volumes are merged into a storage pool, from which logical volumes can be allocated. The TH-VSS supports concatenation (linear), stripe (RAID-0) and mirror (RAID-1) schemes.

In commercial data storage environments, various kinds of storage systems can be attached to the SAN, including the JBOD system, the RAID subsystem, tape drives and storage servers. Different kinds of storage systems have different properties in bandwidth, latency, and reliability. When the meta server starts up, it scans all the storage devices attached to the SAN and writes a label and a UUID at the head of each detected device to mark it as a physical volume. Then it puts the physical volume into the appropriate storage pool according to its property. The composition of a storage pool can be changed dynamically. When a new storage device is added to the SAN, the system monitor on the meta server will detect the addition of the new device, query its type, create a physical volume on it and put it into the appropriate storage pool.

When a physical volume is added to a striped storage pool, the data of the logical volumes must be re-striped among physical volumes to improve performance. A mirroring mechanism is used to ensure that the data of the logical volumes is not corrupted during the re-striping process. Fig. 3 shows the process of re-striping. Data movement can be interrupted at any time due to errors or other reasons. LV1 can continue to function normally because it contains the newest copy of data. 


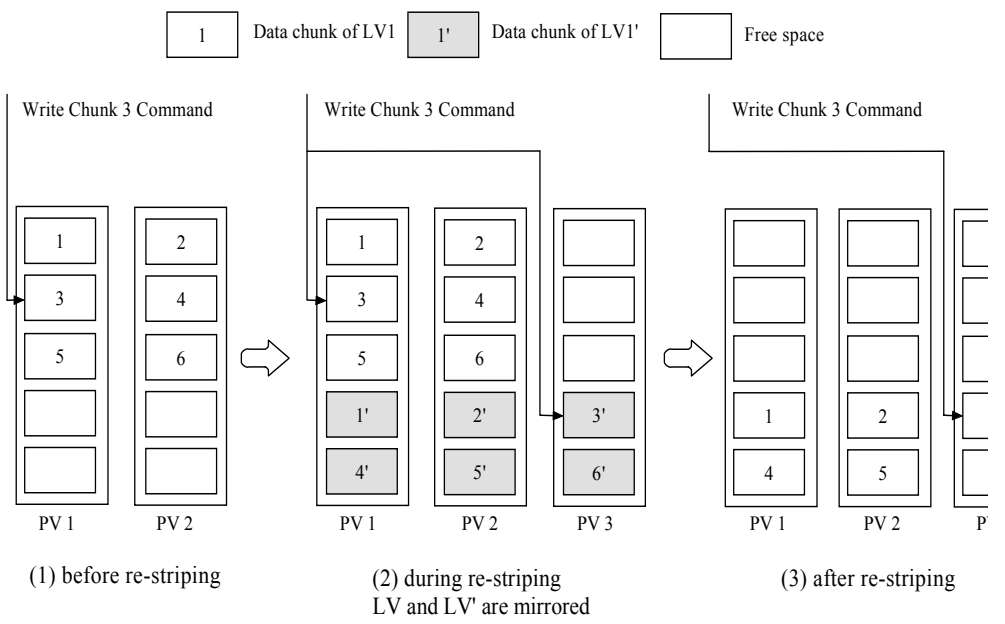

Fig. 3. Process of re-striping

\subsection{Online Change of Mapping Table}

In order to meet the requirements for uninterrupted service, some management tasks need to change mapping tables of logical volumes online. In SAN environments, where storage is shared among multiple hosts, the access of hosts to logical volumes must be controlled so that the data of logical volumes is consistent. A locking mechanism is used to ensure data consistency when the mapping table of logical volume is changed. A meta server may send lock and unlock requests of a particular logical volume to the agent. Upon the agent's receiving a lock request, any I/O request that has already been mapped by the mapping table of a logical volume but has not yet completed will be flushed. Any subsequent $\mathrm{I} / \mathrm{O}$ request to that logical volume will be postponed for as long as the volume is locked. On receiving an unlock request, any postponed I/O request will be mapped by the new mapping table and gets re-queued for processing.

The process by which mapping tables are changed online is as follows. First, the meta server identifies the hosts to which this logical volume has been assigned, and sends a LOCK_LV request to agents on these hosts. On receiving the request, the agent performs the locking operation and then sends a LOCK_LV response to the meta server. After receiving the responses from the agents to the LOCK_LV request, the metadata manager generates a new mapping table. Then it sends a RELOAD_TABLE request to the agents. The agents replace their old mapping tables of the logical volume with the new ones and send a response to the meta server. After all the old mapping tables are replaced with the new ones, the meta server sends an UNLOCK_LV request to the agents. The agents map the postponed $\mathrm{I} / \mathrm{O}$ requests with the new mapping table for processing and send an UNLOCK_LV response. Finally, the metadata manager of the meta server writes the updated metadata back to the heads of the physical volumes. 
In this process, data inconsistency due to hosts' access to a logical volume with different mapping tables is avoided by having the meta server send an UNLOCK_LV request after all the old mapping tables have been replaced with new ones successfully.

\section{Experimental Results}

In this section we will present the experimental results of our virtualization system. The test system consisted of two Linux servers, two Windows servers, a meta server and an FC disk array, all of which were connected to a 2 Gigabit FC switch. Their configurations are shown in Table 1.

Table 1. Test configuration

\begin{tabular}{llll}
\hline Machine Linux server & Windows server & Meta server \\
\hline CPU & Intel Xeon 2.4GHz x 2 & Intel Itanium2 1GHz x 2 & Intel Xeon 2.4GHz x 2 \\
Memory & 1GB & 1GB & 1GB \\
OS & Linux(kernel: 2.4.26) & Windows Server 2003 & Linux(kernel: 2.4.26) \\
FC HBA Emulex LP982(2Gb/s) & Emulex LP982(2Gb/s) & Emulex LP9802 \\
FC Disk & Seagate ST3146807FC x 5 & \\
\hline
\end{tabular}

\subsection{Overhead of Virtualization Layer}

We used the Intel Company's IOMeter test program to evaluate the overhead introduced by the virtualization layer. First we tested the performance of a plain FC disk. Then we created a linear storage group on the disk and allocated a logical volume from it. To derive the overhead, we compared the performance of the VSS volume to the performance of the physical disk. The access pattern was sequential reading in 4 -KB blocks. The comparison of average response times for different block sizes is shown in Fig. 4.

We can see from the results that the impact of the virtualization layer on the $\mathrm{I} / \mathrm{O}$ performance was negligible in respect to bandwidth and average response time.

\subsection{Impact of Stripe Mapping Strategy}

In order to evaluate the performance improvement when using striped storage pools, first we created a striped pool with one disk and allocated a logical volume LV1 from it. We tested the performance of LV1 using Bonnie Benchmark. We created a file system on LV1 and allowed random access to fixed size files. We measured the number of random seeks per second on the LV1. Then we added another disk to the pool and repeated the test. The process was repeated until 


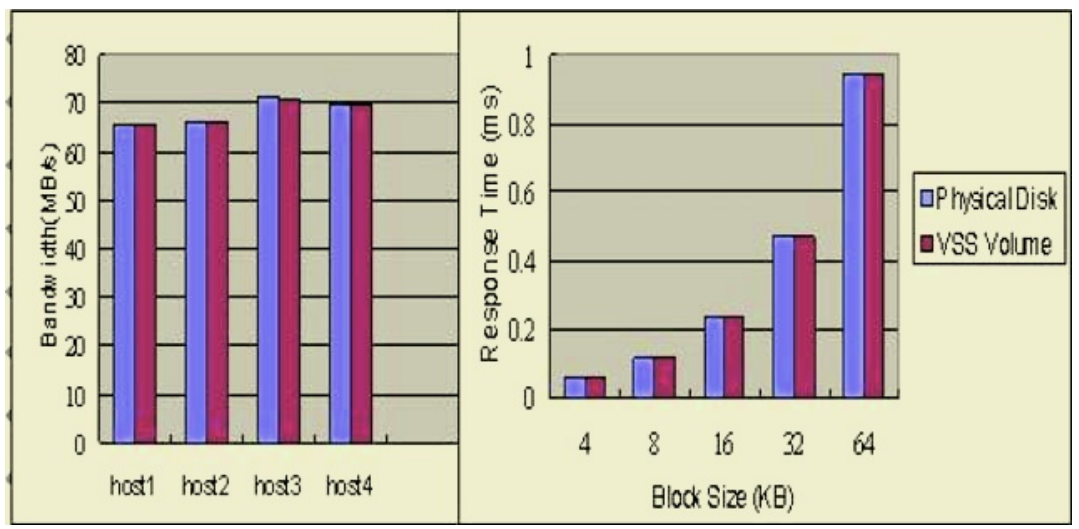

Fig. 4. Overhead of TH-VSS. First figure shows the comparison of bandwidth. Second figure shows the comparison of average response time

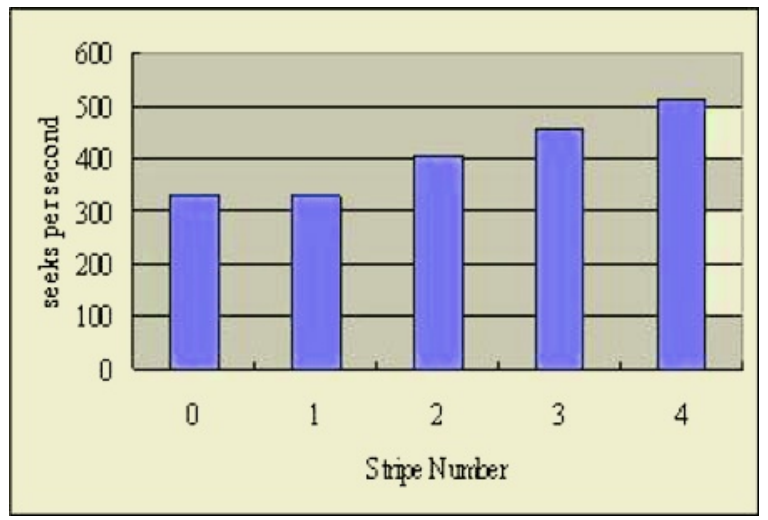

Fig. 5. Impact of stripe mapping strategy on performance

four disks had been added to the pool. The results are shown in Fig. 5, including a comparison of the results with the case of a plain disk labeled with 0 .

The figure shows that the striped logical volume can provide better I/O capacity than that of a single disk, and the more disks in the striped storage pool, the better the performance of the LV1. When the LV1 is striped over 4 disks, the number of seeks per second is increased by $55.2 \%$.

\section{Conclusion}

In this study, we designed and implemented a storage virtualization system for the SAN environment. An asymmetric architecture was adopted to achieve a 
single point of management, and metadata consistency was ensured in the SAN environment where storage is shared by multiple hosts. The new system's meta server implements the function of dynamic management of storage resources and system monitoring. Storage pools with different properties can be provided. The test results showed that the addition of our virtualization layer introduces little overhead to the system, and the striped storage pool can improve I/O performance effectively.

\section{Acknowledgements}

The work described in this paper was supported by the National Natural Science Foundation of China (No. 60473101) and the National High-Tech Research and Development Plan of China (No. 2004AA111120).

\section{References}

1. David, T., Heinz, M.: Volume Managers in Linux. In Proceedings of the FREENIX Track:2001 USENIX Annual Technical Conference, Boston, Massachusetts, USA (2001)

2. Sistina Software, Inc.: Logical Volume Manager. http://www.sistina.com

3. Heinz, M.: Linux Cluster Logical Volume Manager. In Proceedings of the 11th Linux Kongress , Erlangen, Germany (2004)

4. Hewlett-Packard Company: HP StorageApps sv3000 White Paper. (2002)

5. Ram, P.: EVMS Cluster Design Document. http://evms.sourceforge.net/clustering/

6. SAN Symphony version 5 datasheet. http://www.datacore.com (2002)

7. StoreAge White Paper. High-Performance Storage Virtualization Architecture. http://www.storeage.com

8. André, B., Michael, H.: V:Drive - Costs and Benefits of an Out-of-Band Storage Virtualization System. In Proceedings of the 12th NASA Goddard, 21st IEEE Conference on Mass Storage Systems and Technologies, College Park, Maryland, USA (2004)

9. Chang Soo Kim, Gyoung Bae Kim, Bum Joo Shin: Volume Management in SAN Environment. In Proceedings of 2001 International Conference on Parallel And Distributed Systems, KyongJu City, Korea (2001)

10. Common Information Model (CIM) Specification, v2.2. http://www.dmtf.org/ standards/cim

11. Friedhelm, S.: The SCSI Bus \& D E Interface. Addison-Wesley, second edition (1998) 